\title{
An Integrated System for Communication and Equipments Control Using Radio Link
}

\author{
Alberto Tronconi, Marco Billi \\ Consiglio Nazionale delle Ricerche \\ Istituto di Ricerca sulle Onde Elettromagnetiche \\ Firenze, Italy
}

\begin{abstract}
In the following we will describe a PC based system which allows motor disabled persons to control devices in a domestic environment. The system will be able to "learn" and reproduce infrared (IR) code from virtually every IR controller currently available, so it can relay on a wide range of commercial devices, now and in the future. The configuration phase will be quite easy, enabling non-experts to perform, without training, the operation needed to adapt the system to user needs. The control code will be sent via a radio link, to allow disabled users to control the desired device without moving around to "see" the receiver. The disabled user will interact with the environment via a highly adaptable iconic interface, designed to be used either to control devices or to send messages to the surrounding environment or to a remote supervising station.
\end{abstract}

\section{Introduction}

Disabled people who are unable to control their movements are often unable to use a number of devices in the domestic or working environment, and in some cases even to communicate basic needs or desires.

Our experience in developing communication systems for motor disabled people has lead us to consider the opportunity of using one of the interfaces developed which allows users to control devices in their living environment as well as communicate. We integrated the two features in a unique system capable of acting as a highly intuitive communicator, to send messages either to the local environment or to a remote supervising station and as a means to control a number of devices.

The control system has been designed to comply with three main requirements: to work with a wide range of commercial product now and in the future, to let users operate as freely as possible in a domestic environment, and to interact with the system in a straightforward way.

To achieve these results, we relied on our communication system (MACDIP [1]), which offers a good foundation in terms of flexibility and effectiveness, providing a multimedia editor capable of dea:ing with photographic image and sound in a friendly way with a highly adaptable and straightforward user interface. 


\section{The Communication System}

We have chosen to assume, as base module, the system named MACDIP (an acronym for Multimedia Ambiental Communication sy stem for DIsabled People) for interfacing and communication, both for its straightforward interaction method and its adaptability to user needs.

The communication process, adopted by MACDIP, consists of selecting an ichnographic representation of the desired issue, causing a message to be emitted to the surrounding environment in vocal form and/or sent to a remote control station.

In order to adapt the system to user needs, operators can rely on a multimedia editor to compose a communication pattern, using multimedia elements like photographic images and digital sound. Operators can grab and choose suitable graphic or photographic images from within the multimedia editor, as well as compose the desired images sequence and link messages to every image into a friendly environment, without specific knowledge.

Furthermore, operators can choose from a number of special input devices and the interaction method to comply with the user's residual skills.

The resulting communication environment will be highly customized, making the recognition process and the interaction with the system as easy as possible.

Relying on the above mentioned features, we added the possibility to the multimedia editor of joining commands to devices to the images while composing the communication pattern. In such a way the user will then be able to control the surrounding environment by choosing the image which corresponds to the desired action, or to send a local or remote message.

\section{Structure of the Control System}

The control system will consist of hardware and software tools to record useful control codes from different IR controllers, associate a unique identifier to each code, and store them in a file waiting to be used.

While composing the interface, which is done by means of the previously mentioned multimedia editor, the identifier of the desired control code will be joined to a representative image.

When the user selects the icon related to an action, the suitable control code will be retrieved by means of the associated identifier and routed, via the serial port, to a transmitter that sends the code to activate the desired equipment.

In order to overcome restrictions, due to the IR's impossibility to trespass solid object, the transmission relies on a radio link going from the control station to one of the repeaters placed in suitable places throughout the house. The repeater will transform the received code in the original IR signal to activate the target equipment.

\section{Recording Commands}

One of the main issues we tried to fulfill involves the possibility of employing the 
control system independent of specific types of controllable devices, so that the user is free to choose the best suited brand or type of controlled equipment.

At present, the most common methods for controlling remote devices in a domestic environment are based on infrared (IR) controllers. Nearly all common electronic equipment (TV set, CD player, etc.) can be handled via an IR controller. The same method is now used to control lights, heating, and other common devices.

Moreover, a number of devices exist, which have been specifically developed for disabled people (e.g. to open/close doors and windows, to handle a telephone, to turn on and off electronic equipments), that can even be handled by an IR controller.

The combination of the wide availability of IR controlled devices and the fact that users are free to use the equipment that best suits their needs led us to design a system that could interact with unknown heterogeneous controlled equipments, using the IR link. Unfortunately an industrial standard on the format of IR commands does not exist, so that they cannot be prestored, but must be recorded by the user.

To solve the problem, we provided our system with a suitable software tool that could control, via the serial port, an IR receiver. The receiver samples an IR control signal and sends the result to the software tool, which stores it in a file. The sampled code will be combined with an identifier (a string of maximum 32 alphanumeric character), the number of target repeaters (explained below), which the code will be sent to, and the duration of the signal in milliseconds.

The user can record all the desired control codes, which can be edited or updated every time $\mathrm{s} / \mathrm{he}$ desires.

Inside the multimedia editor, a representative icon will be linked to a specific control code identifier instead of a message, so that the chosen icon when selected will be used to perform the corresponding action.

\section{Radio Link}

Another key issue in developing the control system has been to let users operate in a domestic environment as freely as possible. Since the IR control cannot operate when solid barriers lay between receiver and transmitter, the user would move around to find the right position for activating the chosen device.

This can be difficult or even impossible for people with motor handicaps, so we decided to use a radio link, which is capable of reaching every point of a house, to convey the control code.

This choice would eliminate the purpose of using non-modified commercial equipment, which would require special controlled devices (equipped with radio receiver), so we decided to rely on the radio channel to convey commands and on an IR link to activate the target device.

To achieve such a compromise, we developed an hardware device composed of a radio receiver and transmitter and an IR transmitter, which will act as a repeater, translating the code from the radio to the IR form.

A suitable number of repeaters will be placed in such a way as allow direct control of all the desired equipment, therefore giving the disabled user the ability to 
control all equipment, regardless of his/her position.

When an user chooses a command the developed software will send it, via a serial port, to an other hardware device (master), that delivers the command via a specific radio protocol to the right repeater. Then the master returns an appropriate code, depending on the success or failure of the command delivery.

\section{Radio Protocol}

Since in some cases the user could not directly see the result of expected action and since the result of an action can be uncertain when relying on a radio link (eventually affected by noise), we developec a simple protocol to assure the success of IR code delivery.

First of all, when a code has to be transmitted, it is related to the identifier of a specific repeater. Then, the master will send an attention signal, waiting for an acknowledgment. The transmitted signal contains the identifier of the target repeater and that of the transmitting station. If the acknowledgment is not received in $200 \mathrm{~ms}$, the attention signal will be sent again, three times at most. If the replay of the target repeater is lacking, an error message will be displayed to inform the user that a specific repeater does not respond.

In the ordinary operation, the repeater replies with the acknowledgment signals, then the master sends another signal containing the repeater identifier, the selected code and the transmitting station ID. The code will be sent back by the receiver to control the correctness of the transmission. If the code is wrong, it will be sent again until it is received correctly; otherwise a terminating signal will terminate the transaction. At this point the repeater will send the code, in IR form, to the target receiver.

This protocol will assure high error immunity and reduce interference in environments where more than a single user is employing the control system; at present we are developing a more complete protocol to handle collisions.

\section{Feedback}

In the composition phase, the operator can choose if and how the user must be informed of the progress in code delivery. This can be done using symbolic, text, or phonic messages which state the correctness of a transaction or the type of error incurred.

In some cases, the user has to be aware of what is happening when the system performs an action, even if it will take place in a hidden location. The system does not presently integrate any method to allow direct monitoring of hidden equipment; a common solution can be to use closed loop TV equipment, eventually controlled by our system. 


\section{Software and Hardware Tolls}

As mentioned before, the control system requires software tools to control the recording and delivering phases, and hardware tools to sample IR codes, as well as transmit and receive codes between the control station and a repeater.

We developed the software tool using the Microsoft Visual $\mathrm{C}++1.0$, the natural environment of these tools is MS Windows, but the basic communication routine can be used even by software running under DOS.

This feature allows us to maintain the compatibility with the existing DOS systems, like MACDIP, but to be ready for future developments under MS Windows.

The implemented tools have been designed to be as friendly as possible, using online help and hints to guide the novice user. As result a very short training period is required: our experience has shown that a two-hour training session is sufficient for an adequate knowledge of the entire system (communication and control).

Hardware tools consist of the IR sampler to capture the IR code, the master, the radio receiver and transmitter, atd the repeater. The radio equipment is commercial, while other features of the master and repeater are based on an 8 bit microcontroller (by SGS), which contain the microcode to perform the described protocol and implement the IR receiver and transmitter.

The microcontroller exchanges data with a radio modem, integrated in the circuitry, via a serial connection at 1200 baud; the modem perform an FSK modulation/demodulation to send/receive information to/from the external radio equipment

\section{Conclusion}

The outcome of our effort is a system that integrates the possibility of controlling a wide range of equipments and communicating simple messages to the local environment in a phonic form, or to a remote supervising station via a radio link.

Both the performed functions (communication and control) will be highly customizable by means of some software tools that allow easy adaptation of the system to user needs on the one hand, and to the controlled equipment and output devices on the other.

Initial laboratory tests show us that the result is an easy to use and effective system that can help people with motor disabilities to better interact with their living environments, without constraints on the type of controlled equipments or the activating process, while requiring a few hardware apparatus installed in the controllable environments.

\section{References}

1. A. Tronconi, R. Tronconi, M. Billi, L. Stefani, F. Focardi: A system for basic communication aimed at severe phisically disbled people. Proceedings of $3 \mathrm{rd}$ International Conference on Computers for Handicapped Persons, Vienna, July 7-9, 1992 
2. ESPRIT 2431, Home system specification, Commission of the European Communities, DG XIII, 1991

3. TIDE 2nd phase Workplan Draft, Commission of the European Communities, DG XIII, 1992

4. C. Bühler: Uniform user interface for communication and control. Proceedings of the 2nd European Conference on the Advancement of Rehabilitation Technology, May 26-28, 1993 Stockholm, Sweden.

5. R. Bianchi Bandinelli: L'edificio intelligente: stato dell'arte e futuri sviluppi. Atti del $3^{\circ}$ Convegno Nazionale "Informatica Didattica e Disabilità", Torino 4-6 Novembre 1993. 